\title{
Starting Point, Keys and Milestones of a Computer Code for the Simulation of the Behaviour of a Nuclear Fuel Rod
}

\author{
Armando C. Marino ${ }^{1,2}$ \\ ${ }^{1}$ Comisión Nacional de Energía Atómica (CNEA), Centro Atómico Bariloche (CAB), Bariloche, Río Negro, Argentina \\ ${ }^{2}$ Instituto Balseiro, Universidad Nacional de Cuyo, Bariloche, Río Negro, Argentina
}

Correspondence should be addressed to Armando C. Marino, marino@cab.cnea.gov.ar

Received 24 August 2010; Accepted 19 November 2010

Academic Editor: Juan Pablo Ordóñez

Copyright (C) 2011 Armando C. Marino. This is an open access article distributed under the Creative Commons Attribution License, which permits unrestricted use, distribution, and reproduction in any medium, provided the original work is properly cited.

The BaCo code ("Barra Combustible") was developed at the Atomic Energy National Commission of Argentina (CNEA) for the simulation of nuclear fuel rod behaviour under irradiation conditions. We present in this paper a brief description of the code and the strategy used for the development, improvement, enhancement, and validation of a BaCo during the last 30 years. "Extreme case analysis", parametric (or sensitivity), probabilistic (or statistic) analysis plus the analysis of the fuel performance (full core analysis) are the tools developed in the structure of $\mathrm{BaCo}$ in order to improve the understanding of the burnup extension in the Atucha I NPP, and the design of advanced fuel elements as CARA and CAREM. The 3D additional tools of BaCo can enhance the understanding of the fuel rod behaviour, the fuel design, and the safety margins. The modular structure of the BaCo code and its detailed coupling of thermo-mechanical and irradiation-induced phenomena make it a powerful tool for the prediction of the influence of material properties on the fuel rod performance and integrity.

\section{Introduction}

The BaCo code (BaCo means "Barra Combustible", Spanish expression of "Fuel Rod") was developed at the Atomic Energy National Commission of Argentina (CNEA, "Comisión Nacional de Energía Atómica"). The development started at the end of the 70's in order to cover the study of the fuel rod behaviour under irradiation conditions [1]. The first studies were the analysis of the relative influence of the modelling in the simulation of the fuel rod behaviour [2].

At present $\mathrm{BaCo}$ is part of the design of advanced PHWR $[3,4]$ and innovative PWR $[5,6]$ fuels. Likewise we are participating in the CRP FUMEX II of the IAEA (Coordinated Research Project on Fuel Modelling at Extended Burnup II of the International Atomic Energy Agency) [7] among other tasks including teaching activities.

At the beginning of the development we defined a clear strategy and some simple rules in order to keep the coherence and modular structure of the BaCo code. The adopted strategy facilitated performing changes to the model, the incorporation of new issues to fuel behaviour, new calculation options, and integrating alternative models.
In this paper we present the strategy for development, improvement, enhancement, and validation of BaCo. We illustrate the different steps of the development by using selected examples where our code was extremely important. We shall expand the analysis of fuel performance in Section 3, the fuel rod design in Section 4, and the additional tools of $\mathrm{BaCo}$ in Section 5.

\section{The BaCo Code}

2.1. Briefings about BaCo. The BaCo code structure and models have already been described in [8], including steady state and transient thermal analysis. Nowadays, the number of instructions is about twelve thousand FORTRAN 90 lines. Data postprocessing and the integration with $3 \mathrm{D}$ calculation using finite elements improve the output of $\mathrm{BaCo}$ and the analysis of results. The last versions of the code were developed at the DAEE Division ("División Diseños Avanzados y Evaluación Económica"-Advanced Design and Economical Assessment Division, CAB, CNEA). We participated in several international programs concerning comparisons between codes and experiments $[7,9,10]$. 
At present we are collaborating in the Coordinated Research Project on Fuel Modelling at Extended Burnup II organized by the International Atomic Energy Agency (IAEA) [7].

The $\mathrm{BaCo}$ modelling of $\mathrm{UO}_{2}$ pellets includes elastic deformation, thermal expansion, creep, swelling, densification, restructuring, relocation, cracks and fission gas release. For the Zry cladding, the code models elastic deformation, thermal expansion, anisotropic plastic deformation, creep and growth under irradiation. The modular structure of the code easily allows us to input different material properties. It can be used for any geometrical dimension of cylindrical fuel rods with $\mathrm{UO}_{2}$ (or MOX) pellets (either compact or hollow, with or without dishing) and Zry cladding.

A special feature of the $\mathrm{BaCo}$ code is its complete treatment of the fuel with or without mechanical contact to the pellet surface and the clad, at any irradiation stage. It can model self-standing clad sheets (like LWR and Atucha fuel rods $[11,12]$ ) or collapsible clad sheets (like CANDU fuel rods [13]), without special changes in the physical models triggered by the user input. $\mathrm{BaCo}$ includes excellent compatibility for PWR [9], BWR [9], WWR [7, 14], MOX $[15]$ and also experimental fuels $[7,9]$.

Fuel rod power history and either cladding or coolant external temperatures must be given to the program. Rod performance is numerically simulated using finite time steps (finite differences scheme). The code automatically selects time steps according to physical criteria. Temperature profiles within pellet and cladding, main stresses at pellet and cladding, radial and axial crack pattern in the pellet, main strains and hot geometry of pellet and cladding, change in porosity, grain size and restructuring of the pellet, fission gas release to the free volume in the rod, trapped gas distribution in the fuel and in the $\mathrm{UO}_{2}$ grain boundary, internal gas pressure and current composition of the internal gas and dishing shape evolution, are calculated. The output contains the distribution along the rod axis of these variables. The details of the mechanical and thermal treatment and the pellet, cladding and constitutive equations are available in [8] and a brief description of the code is included in [16].

Nevertheless, the brief description of the code mentioned above is the result of an extensive work where several issues were taken into account. Some of those issues were as follows:

(i) the choice of the language (FORTRAN, C, java, etc.), compiler, software tools for postprocessing, operative system, hardware (microprocessor and platform), and so forth,

(ii) the selection of the system of units to be used in the code $\left(\mathrm{MWd} /\right.$ tonUO 2 or $\mathrm{MWd} /$ tonU, ${ }^{\circ} \mathrm{C}$ or $\mathrm{K}$, etc. $)$,

(iii) the definition of a roadmap with several stages in order to focus the development on particular objectives (or milestones),

(iv) an identification of the calculation requirements (for $\mathrm{BaCo}$ it was PHWR at the beginning and, later on, PHWR \& PWR),

(v) the selection of the main attributes of fuel rod behaviour modelling to be included in the code, (vi) the arrangement of a fundamental database of irradiation including the main fuels of interest for CNEA.

2.2. Strategy and Milestones of a Fuel Code. The development of such a complex tool as a code for the simulation of nuclear fuel rods comprises several steps [17]. We defined three main steps for the BaCo code. Figure 1 is a roadmap for the development and continuous improvement of $\mathrm{BaCo}$. The plot shows the concatenation of the three steps, each of them composed by substeps. Some of these substeps could modify an earlier step in the subsequent loop.

The first step is the tuning of the mathematical procedures, the fuel behaviour models and a narrow set of selected experiments. This process includes the following:

(i) the outline of the problem to solve;

(ii) the mathematical approach to the problem. An algorithm is defined enabling the problem to be solved by an analytical procedure or numerically;

(iii) testing the method's convergence;

(iv) the selection of the behaviour features that will be included in the code, by selecting the most representative physical models. Usually, the procedure starts including simple features as elasticity and thermal behaviour and progressively adding new ones in subsequent loops with a new substep inside the first step. The procedure takes into account that modelling changes could modify the mathematical approach (i). The first models included in the BaCo code were from the EPRI [18], MATPRO [19] and Olander [20]. The flux diagram included in Figure 2 shows the important interactions existing among different characteristics of fuel rod behaviour. Each block includes at least one model of fuel behaviour and this is the starting point of the code programming. Usually each item of the diagram is associated to a subroutine or a couple of subroutines in the program. An initial version of the code can be written without taking into account all the items. However, considering in advance the inclusion of all the behaviour characteristics in the flux diagram of the code facilitates the programming of new blocks and the understanding of their relative influence among the already implemented models;

(v) comparison between the code predictions and selected experiments with very well-defined parameters. At this point the concept of simulation algorithm flexibility is introduced in order to accommodate the boundary conditions for each particular case. Depending on the results, this is either the end of the first step or, in case it was needed, the definition of a new first step loop. From this point on, we have a "code".

The procedures (ii) to (iv) constitute a cycle not strictly close because we can go further to the 2nd step concerning applications where the code is applied to technological 
outstanding situations. The possibility to pass from the 1ststep to the 2nd step depends on successfully completing the procedures from (i) to $(\mathrm{v})$ at least once, taking into account that the mathematical coherence of the code was established. In addition, an adequate user's manual with the documentation of models, approaches and computer procedures must be provided. Important results can be found at the end of the first step as the relative influence of the different models used in the code.

When the task involving procedures (ii) to (iv) is a success, then point $(\mathrm{v})$ can include the participation on international benchmarking of fuel codes in order to test the accuracy of the calculation results. Those tests are a set of exercises with the participation of different codes of several countries. We compare the performance of an individual code against experimental results plus the PIE and/or the results of the other participants. The cases to simulate are as follows:

(a) blind exercises where the user does not know the experimental performance of the fuel rod, as it was in the CRP FUMEX I [9], the evaluation of the EPRI [18] and the D-COM [21],

(b) exercises where we know the experimental results, as in the CRP FUMEX II [7],

(c) idealized or very simplified experiments for code comparison where the main idea is the analysis of the relative influence of the models and the fuel parameters, as in the CRP FUMEX I and II.

Point (v) of the roadmap is the beginning of the second step when we start with the practical application of the code. Here we can:

(vi) assist in the definition of the experimental parameters of fuel rod irradiation. BaCo participated in the irradiation of our own first PHWR MOX fuel, irradiated at the Petten reactor, Netherlands [15],

(vii) analyze the performance of commercial fuel rods and verify the fuel design (as we presented in [4] where we analyzed the SEU ("Slightly Enrichment Uranium") fuels of the Atucha I NPP),

(viii) design innovative fuel elements. BaCo is being used for the design of the CARA fuel [22], a new fuel for our CANDU, Atucha I and II reactors, and the fuel for the CAREM reactor $[5,6]$, an innovative small PWR reactor [23],

(ix) design the experimental irradiations in order to verify new designs of (viii). At present we are defining the irradiation tests requirements of the CARA fuel [24].

Each one of the items of the 2 nd step in the roadmap (Figure 1) could modify the models included in the code or could need a comparison with experiments. Then a new 1st step loop could be produced.

After finishing the 2nd step we can initiate a new loop in order to enhance the code, further extending its field of application. This step is actually necessary when a new challenge is presented. Examples of this situation are the burnup extension of the present PWR or PHWR NPPs and/or the needs of advanced fuels for innovative reactors. The new design could demand new models, reprogramming, additional computer tools and/or to define the experimental support. A new challenge is the simulation of the fuel behaviour with "ab initio" models. Its implementation would strongly extend the applicability of the codes [25], although the coupling between models of Figure 2 could change, making necessary a new formulation of the 1st-step mathematical approach. The result could be a new version of the code with deep changes compared to the original formulation. In such a case, a new loop with the roadmap of Figure 1 has to be started.

A previous step before attempting the development of a new code or a new release could be the programming of "additional tools". Examples are the software package for $3 \mathrm{D}$ calculations $[14,26]$ or the "statistical" improvements for advanced fuel designs $[11,27]$. Here the intention is to prepare the new models and/or subroutines but not coupled at all in the original code in order to test the convergence and the weak and strong points of the development.

2.3. Experimental Support. We mentioned in some items of the previous sections the need of experimental support for modelling, calculations and fuel design. This does not mean to perform an "ad hoc" experiment. The experimental support can be provided by:

(a) the open literature,

(b) the results of experiments at the "International Fuel Performance Experiments" (IFPE) database of the "Organization for Economic Co-operation and Development” (OECD) [28],

(c) the participation in international benchmarking as we mentioned in the previous section where the irradiations used for evaluation correspond to very well-selected experiments [7, 9, 18, 21],

(d) participating in international programs of irradiation where we could share the cost of the experiments by the finding of mutual needs,

(e) being a member of an international project of irradiation as the Halden Reactor Project ("HRP") of the OECD.

Finally, the last choice when the information is not available is to perform the experiment. Nevertheless, the code will provide a frame to define the power history and the parameters of the experiment after the analysis of similar irradiations and simulations. This is a useful tool to reduce the number of experiments which, together with the code results, will constitute the complete description of the fuel behaviour.

\section{Fuel Performance Analysis}

The analysis of the performance of commercial fuels begins with the study of normal cases. Afterwards, we continue the analysis with fuels under demanding conditions of 


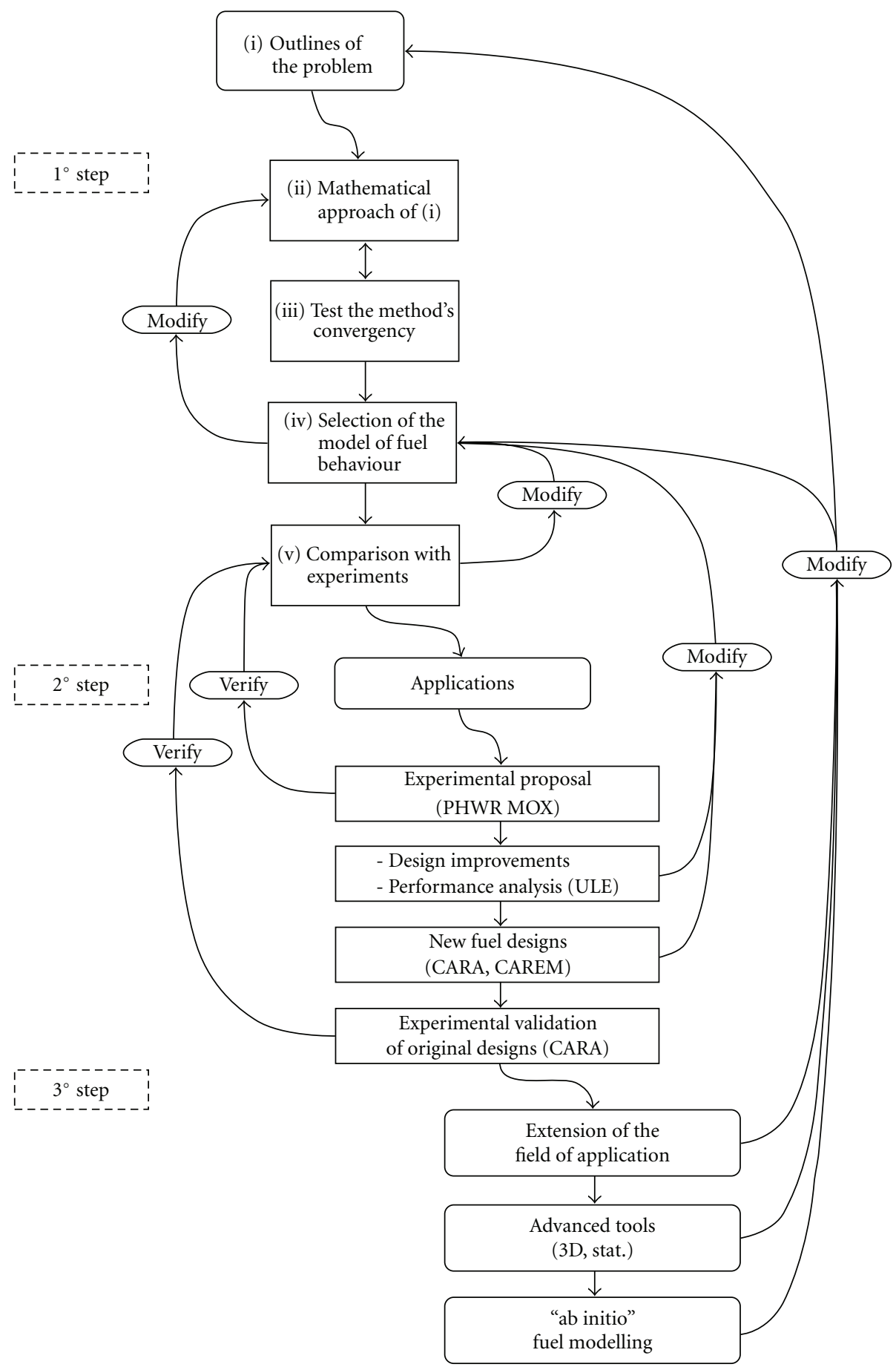

Figure 1: Roadmap of the development of the BaCo code.

operation. The main objectives of these works are the improvement of the fuel design and the review of the management rules for the fuel.

$\mathrm{BaCo}$ allows the calculation of a complete set of irradiations of the same fuel design and the calculation of a complete core. We illustrate the performance analysis with the study of the first SEU core of the Atucha I NPP [4]. Using the detailed power history of all fuel elements,
BaCo was used to calculate fuel behaviour indicators, like stresses, temperatures, dimensions, pressures and gasses releases. Figure 3 presents the maximum hoop stress reached at the inner surface of the cladding for each fuel rod during each individual irradiation. The average hoop stress values increase slightly with burnup and change from $\sim 60$ to $\sim 40 \mathrm{MPa}$ depending on burnup. All the fuel rods are well below the $\sigma_{\mathrm{SCC}}$ value to produce a failure due to 


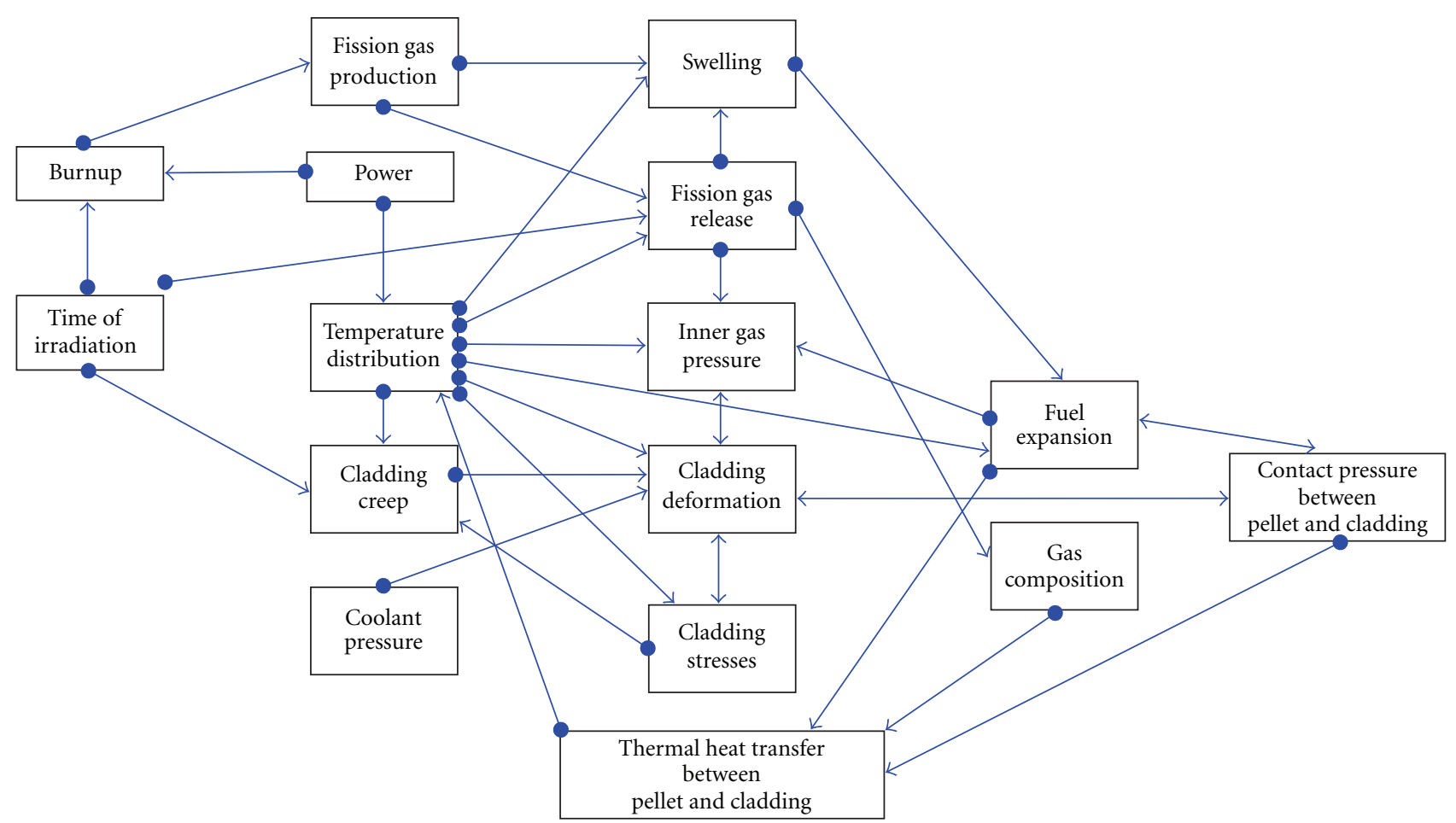

FIGURE 2: Flux diagram showing the strong interaction existing between different characteristics included in the simulation of the fuel rod behaviour.

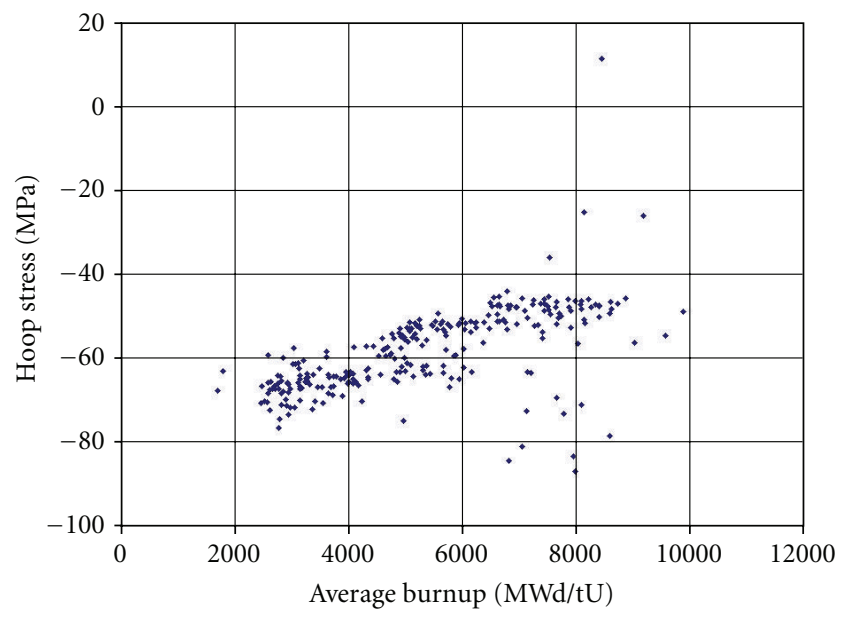

FIGURE 3: Maximum hoop stress at the inner surface of the cladding calculated for each fuel of the first SEU core of the Atucha 1 NPP.

PCISCC $\left(\sigma_{\mathrm{SCC}}=170 \mathrm{MPa}\right)$. Stress reversal occurs only in one fuel rod. The rest of the rods are under compression during the irradiation. Figure 4 includes de maximum pellet temperatures. All the fuel presents a maximum temperature below $\sim 1900^{\circ} \mathrm{C}$ with an average value of $1700^{\circ} \mathrm{C}$ which decreases with burnup. We could expect columnar grains at the pellet centre of the fuel and for a few pellets a small hole. The values are acceptable for Atucha fuel, because it is a relative thick pellet as compared with other self-standing rods like present PWR and BWR.

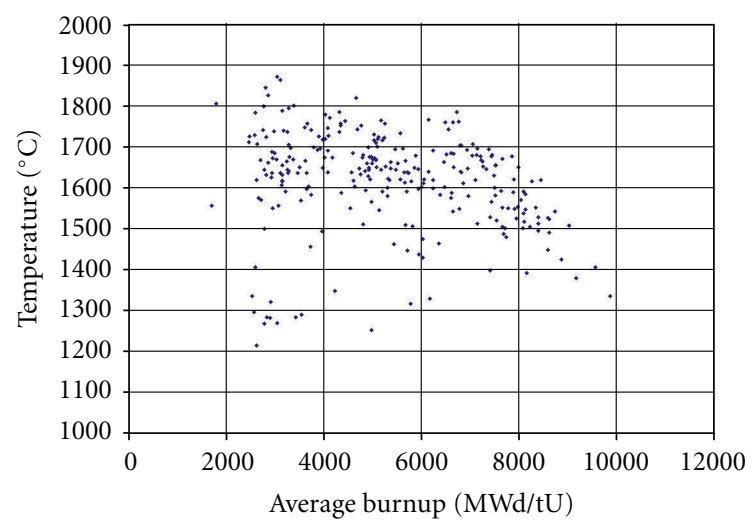

FIGURE 4: Pellet centre temperature for each fuel of the first SEU core of the Atucha 1 NPP.

\section{Fuel Rod Design with BaCo}

Fuel rod design with codes is an analysis performed to find the best parameters to be used in fuel elements in order to improve fuel performance, economics and safety margins. Parameters are the properties of the materials to be used, the nominal geometrical dimensions of fuel rods and the tolerances taken into account in the manufacturing process. We can understand the tolerances as the uncertainties of the nominal values including the statistical dispersion. In order 


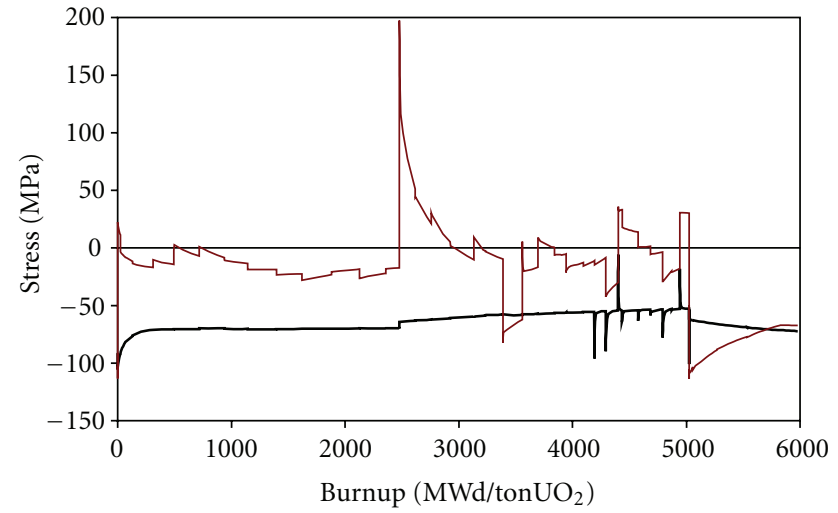

Figure 5: "Hoop stress" at the cladding in the middle of an Atucha I fuel rod with natural $\mathrm{UO}_{2}$. Standard (thick line) and minimum gap situation (thin line).

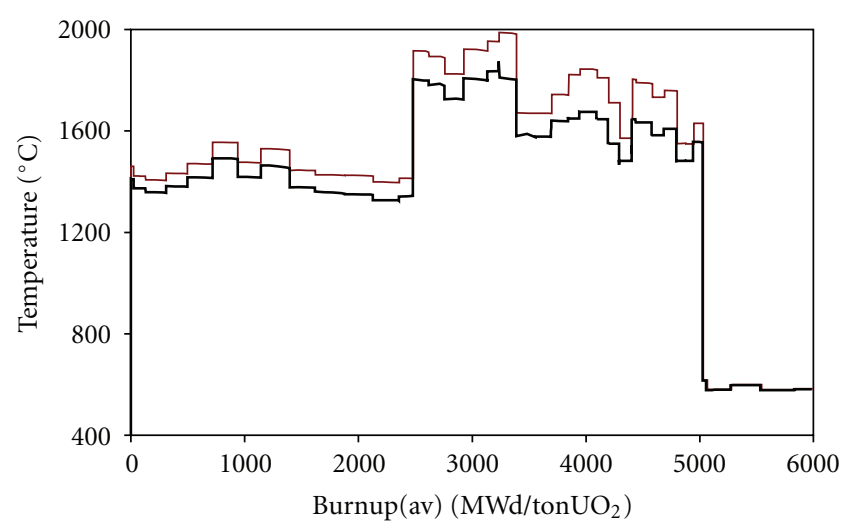

FIGURE 6: Pellet centre temperature during the previous irradiation. Standard (thick line) and maximum gap situation (thin line).

to enhance the fuel rod design we use three different techniques for sensitivity analysis:

(i) extreme cases analysis,

(ii) parametric analysis,

(iii) probabilistic (or statistical) analysis, Those are included in the BaCo code. Nevertheless we must include a last issue:

(iv) the experimental support of the fuel design.

4.1. Extreme Case Analysis. The "extreme cases analysis" consists in finding which combination of fuel rod parameters (code input data) produces the worst situation about fuel rod behaviour. Following this analysis we could define tolerances for fuel rod parameters. This technique is the first step in order to define the as-fabricated tolerances of fuel elements.

The purpose of this analysis is to understand how the combination of assumed extreme rod dimension conditions, within reasonable tolerance limits related to fabrication, can affect the performance (such as maximum stress, maximum

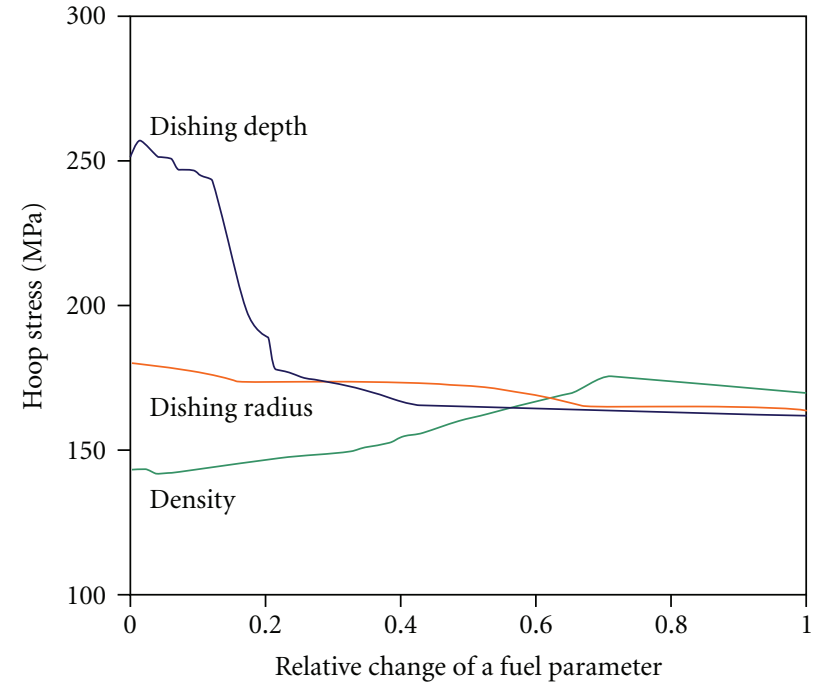

Figure 7: Hoop stress parametric analysis. Analyze of the relative influence of fuel parameters during the first reshuffling of a fuel element irradiated in the Atucha I NPP.

strains, extreme temperatures, etc.). As an example we define two extreme situations:

(i) a rod with the smallest gap between pellet and cladding compatible with the as-fabricated tolerances (see Figure 5),

(ii) a rod with the largest gap (see Figure 6).

The first situation should produce the maximum stress between pellet and cladding, and the second one should result in an increase of the maximum temperature of the fuel.

4.2. Parametric (or Sensitivity) Analysis. The "parametric analysis" is the study of the individual influence of each fuel rod parameter in the fuel rod behaviour (temperatures, stresses, deformations, pressures, etc.). With this analysis we find the correct weight of each fuel rod parameter in order to understand the fuel behaviour with a far and wide scope. This technique is the second step in order to tune the asfabricated tolerances with an engineering overview especially when we are designing fuel elements.

An example of parametric analysis is represented in Figures 7 and 8 . We analyze the relative influence of the dishing parameters of a CARA fuel at the maximum power during the first reshuffling in Atucha I NPP. We are finding the weight of the different rod parameters in order to identify its proper influence on fuel behaviour [27]. Figures only include the most significant parameters of the present calculation: dishing depth, dishing radius, $\mathrm{UO}_{2}$ density, and/or plenum volume. For illustrative purposes we vary the parameters between minimum and maximum reasonable values. Figures 7 and 8 represent the variation between the minimum value of the fuel parameter (0 in the plots) and the maximum value ( 1 in the plots). These reasonable values are the ones obtained from the "extreme cases analysis" and the normal limits of fuel design. The normalized parameters 


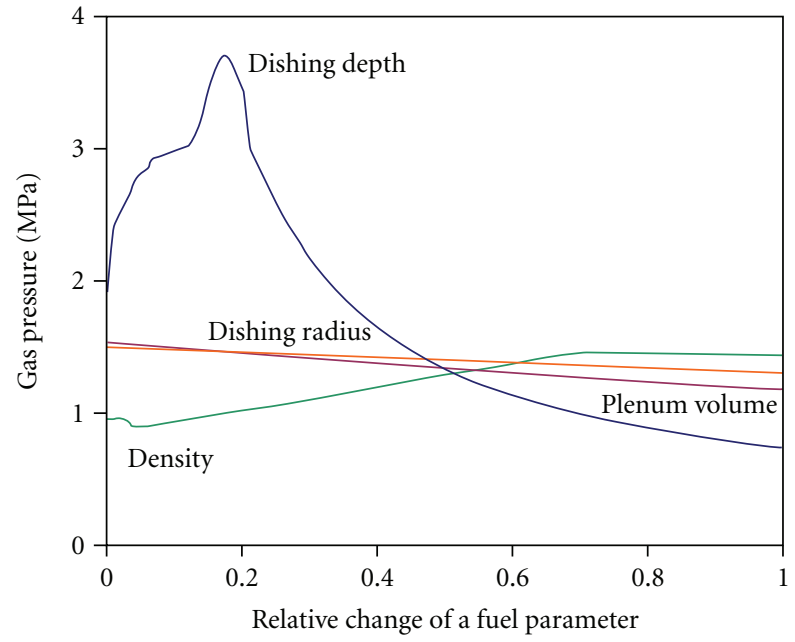

FIGURE 8: Gas pressure parametric analysis. Analyze of the relative influence of fuel parameters during the first reshuffling of a fuel element irradiated in the Atucha I NPP.

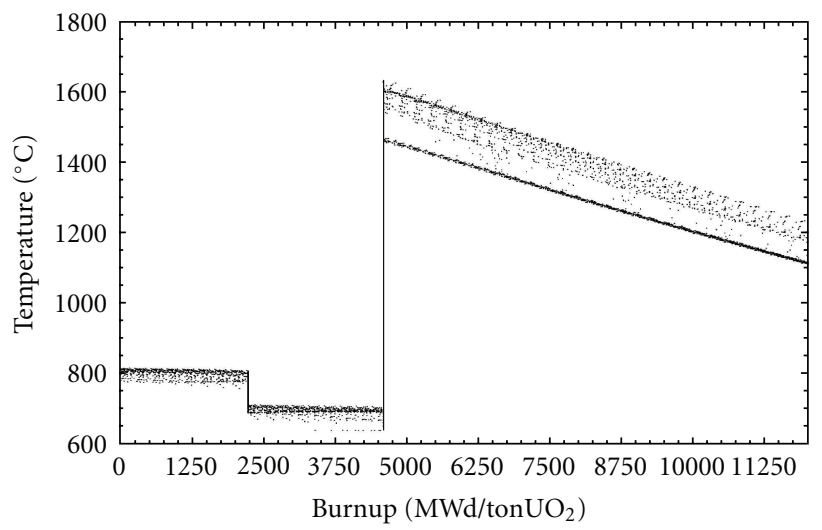

Figure 9: Pellet centre temperature results from the BaCo code probabilistic analysis of a SEU fuel rod of Atucha I (Slightly Enrichment Uranium).

allow us to observe the relative influence of each one in the fuel behaviour. Figures sketch the BaCo results for the hoop stress (see Figure 7) and gas pressure (see Figure 8). We find that the dishing depth is the most relevant fuel parameter in order to reduce the hoop stress at the cladding (see Figure 7). Figure 8 shows the high influence of dishing depth for the gas pressure taking into account its contribution to the free volume, nevertheless the volume of the plenum is associated to the pellet stack and fuel length with a narrow band of variation.

4.3. Probabilistic (or Statistic) Analysis. The "probabilistic analysis" is a MonteCarlo (M-C) technique, which combines several random fuel parameter input data with its statistical distribution. Each probable input data could be a real fuel rod, and the series of MC calculations have a significant impact on the overall calculated results.

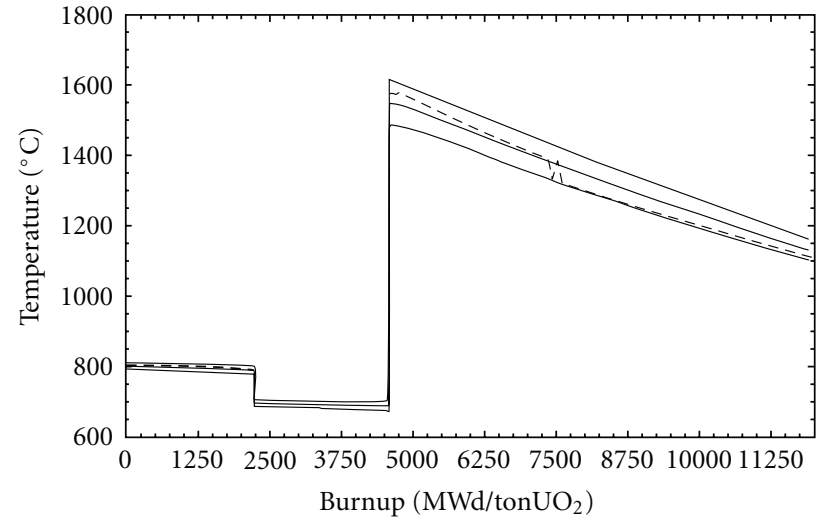

FIgURE 10: Curves with the main values (plus the curves with the main value \pm its standard deviation). The dashed line is the standard calculation with nominal values.

A BaCo's probabilistic analysis of power fuel reshuffling has been performed for the Atucha I NPP [29], where we analyzed the susceptibility of hoop stress during fuel reshuffling at different powers and burnups. Here we reproduce the recommendation of the designer for fuel reshuffling with simplified rules. The fuel for the Atucha I NPP with natural $\mathrm{UO}_{2}$ is analyzed in [11], the same one with SEU is analyzed in [12] and, the fuel for the EMBALSE NPP (CANDU type, Argentina), is analyzed in [13].

As an example of this procedure we calculate the pellet centre temperature of a SEU fuel rod of the Atucha I NPP. The normal calculations are performed using standard values. Nevertheless, the strong demanding conditions during irradiation could produce local failures due to the asfabricated tolerances. The BaCo code allows a probabilistic analysis taking into account the standard values of the fuel parameters with their asfabricated tolerances and their statistical distributions.

Figure 9 presents the result of the probabilistic analysis for the pellet centre temperature. There is a temperature band with less than $200^{\circ} \mathrm{C}$ of uncertainty after reshuffling. This band does not represent a big change in pellet grain morphology and the pattern of cracks. In fact we find two bands, an upper wide band without pellet cladding contact and a narrow band at the bottom of the calculated curves where pellet cladding contact is present. The difference in the width of the bands is due to the uncertainty in the gap size (and the corresponding uncertainty in the gap conductance). Points disappear from the upper band when burnup increases and PCI is done, then the curve jump to the narrow bottom band and the uncertainty in gap conductance is at a minimum. The standard calculation is the result of the $\mathrm{BaCo}$ code when the nominal values of a fuel are used. Note that the standard calculation and the curves with the main values are not the same (see Figure 10). Standard calculation results after fuel reshuffling are equal to the minimum values of the sensitivity analysis results. 
4.4. Experimental Support. The final procedure is the experimental support of the fuel design. Here we identify a few steps:

(a) the weak points of the design,

(b) the most demanding condition of the real operation,

(c) the identification of similar irradiations to our design in the open literature, international programs or institutions,

(d) the simulation with the code of the weak points under demanding conditions,

(e) establishing a set of irradiations in order to check the weak points (a) under the most demanding conditions (b),

(f) the analysis of the irradiation by comparison with our code simulation,

(g) tuning of the code using the new experimental data,

(h) adding the experiment to our database of irradiations (and analyzing whether to share the irradiation with the open database as IFPE).

Nevertheless, the result of the previous step could be that we do not need to perform an irradiation experiment in order to test the fuel rod or at least that the number of irradiations can be strongly reduced.

As an example of the application of the BaCo code in fuel rod design of advanced fuels we present in Figure 11 a ceramography of a PHWR MOX prototype irradiated in the Petten reactor [15]. The preparation of the irradiation, the analyses of the performance and the PIE were made with $\mathrm{BaCo}$. Another example of application of $\mathrm{BaCo}$ is the CARA fuel for the Atucha I NPP (see Figure 12) [22]. This fuel was designed by using the $\mathrm{BaCo}$ code as the main tool following the previous steps described in this section.

\section{BaCo Tools}

5.1. Batch Programming. At this point we mention that the full core performance, the parametric and the probabilistic analyses are the result of careful code programming in order to produce calculations in batches. This simple technique can nevertheless produce valuable information as we presented in Sections 3, 4.2, and 4.3.

5.2. "3D" Enhancement of BaCo. We enhanced the BaCo performance with a complete set of "ad hoc" software package named the MeCom tool. This soft is used for a postprocessing of the $\mathrm{BaCo}$ code calculations. The combination of $\mathrm{BaCo}$, a quasi $2 \mathrm{D}$ code based on a finite differences scheme, and 3D MeCom tools, based on the method of finite elements, constitutes a complete system for 3D analysis of the stressstrain state of nuclear fuel under irradiation [14, $16,22,26]$. The boundary conditions, the initial geometry and the fuel parameters among others are provided for the $\mathrm{BaCo}$ code. The MeCom tools follow the same roadmaps shown in Figures 1 and 2. At the present stage, the models included in MeCom are elasticity, thermal expansion and

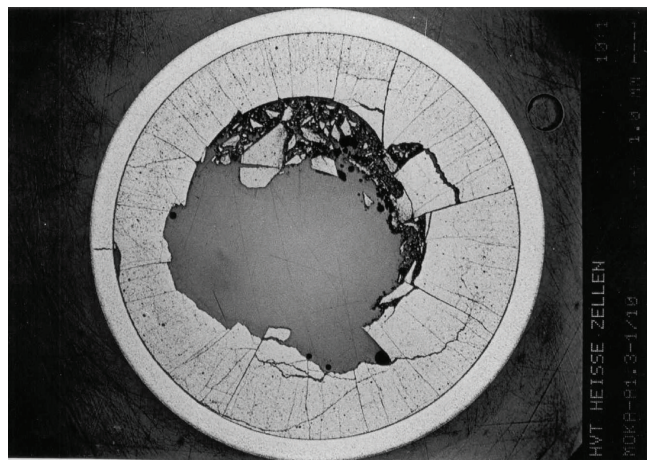

FIgURE 11: PCI-SCC failure of an experimental PHWR fuel rod prototype. The rod was irradiated at the Petten reactor and the experiment was defined with BaCo [15].

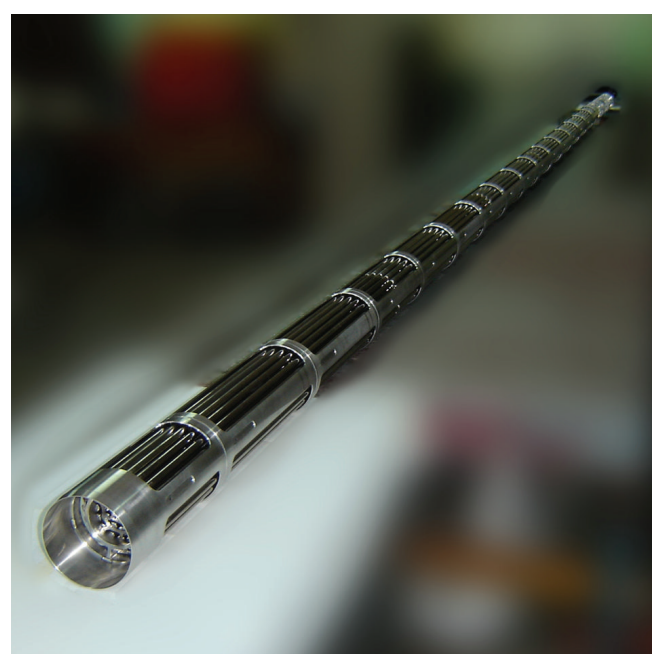

FIgURE 12: First prototype of a CARA fuel for the Atucha I NPP. There are six individual CARA fuels inside a nest made of Zry. The fuel design was assisted with the BaCo code [22].

$\mathrm{UO}_{2}$ cracks. Therefore, the issues presented in Figure 2 are reduced in number. Anyway, they are taken into account in the boundary conditions provided by $\mathrm{BaCo}$.

We find the stresses and the radial profiles of a fuel rod and the shape of the cracked pellet under irradiation showing the bamboo effect and other 3D effects as the presence of the secondary ridge. We mention: (a) the reduction of the deformation of hollow pellets, (b) the absence of ridging when conic shapes are used, (c) the increment of ridging when dishing is present, (d) the reduction of ridging due to chamfers, and (e) the trends of radial deformation by the variation of $l / d$ (height/diameter of the pellet), as interesting examples of $\mathrm{BaCo}+\mathrm{MeCom}$ use. Typically we calculated a pellet ridge height of $\sim 10 \mu \mathrm{m}$ in good agreement with the PWR fuel. Commercial, innovative or unusual pellet shapes can be analyzed by using this package. The results show a good agreement between experimental data and calculations particularly for the pellet radial profile after irradiation. With this tool we enhance the safety margins of the pellet 

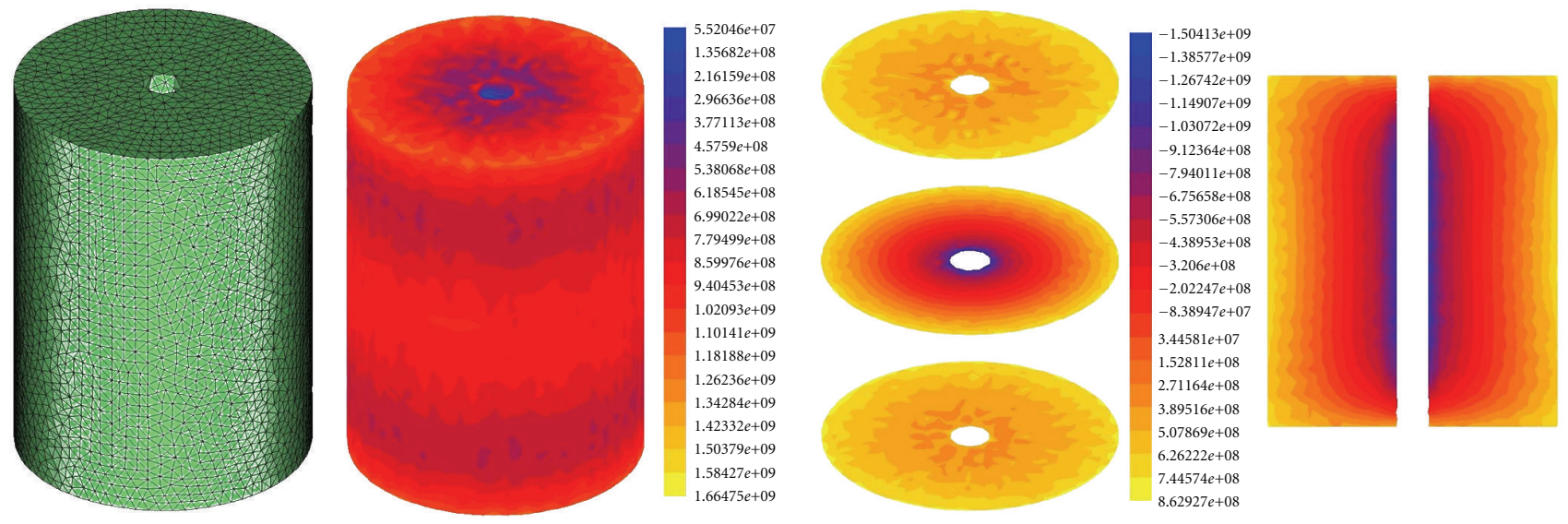

Figure 13: 3D finite elements mesh; von Mises equivalent stresses and tangential stresses (at three different pellet heights and at an axial cut) for a hollowed pellet.
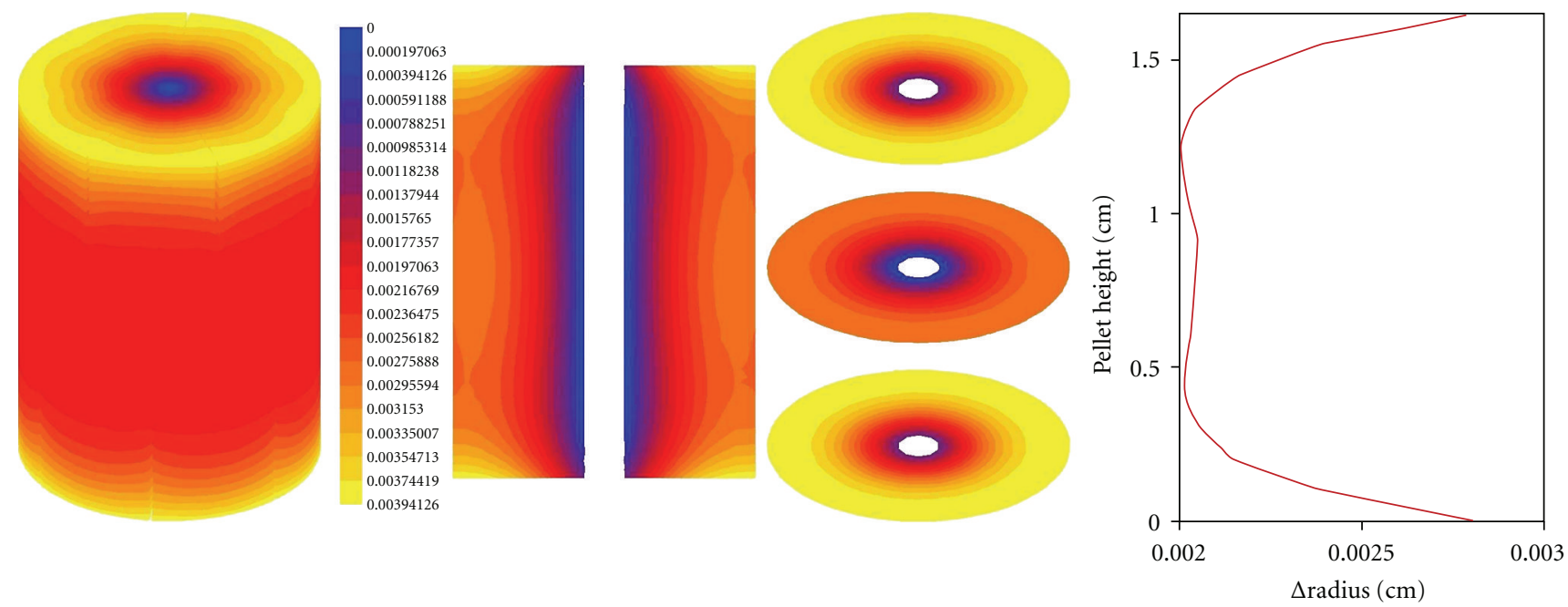

FIGURE 14: Radial deformation of a hollowed pellet from three different points of view and radial deformation.

parameters as shoulder, dishing and central hole among others.

We illustrate our 3D results with the analysis of hollowed pellets as the WWER fuels. Figure 13 shows the mesh for calculation and the corresponding stresses. Figure 14 shows the deformations and the radial profile. The experimental support of this calculation was made by using the data of the Halden Reactor Project and we can find how the presence of the central hole is enough to reduce the radial deformation, the radial stresses, the pellet ridging and the height of the ridges [14].

\section{Conclusions}

The strategy of the development and the continuous enhancement of the $\mathrm{BaCo}$ code were presented together with examples of direct applications in the area of design of advanced nuclear fuels, performance analysis of commercial fuels and 3D enhancement of nuclear fuel modelling. We sketched those issues by agreeing on certain rules at the starting point of code development and by following the roadmaps presented in Figures 1 and 2. These roadmaps helped to clarify the different steps that we had to follow for the development, improvement, enhancement and validation of the BaCo computer code.

"Extreme case analysis", parametric (or sensitivity), probabilistic (or statistic) analysis plus the analysis of the fuel performance (full core analysis) are the tools developed in the structure of $\mathrm{BACO}$ in order to improve the understanding of the burnup extension in the Atucha I NPP and the design of advanced fuel elements as CARA and CAREM. Additionaly, we can understand the relative influence on fuel rod behaviour of the parameters and to enhance the fuel design by using the $\mathrm{BaCo}$ code.

The combination of 3D applications and quasibidimensional codes can enhance the understanding of the fuel rod behaviour as it was shown in the case of the analysis of hollowed pellets. 
The modular structure of the BaCo code and its detailed coupling of thermomechanical and irradiationinduced phenomena make it a powerful tool for the prediction of the influence of material properties on the fuel rod performance and integrity.

\section{References}

[1] S. Harriague, G. Coroli, and E. J. Savino, "BACO (Barra Combustible), a computer code for simulating a reactor fuel rod performance," Nuclear Engineering \& Design, vol. 56, no. 1, pp. 91-103, 1980.

[2] C. Baigorria, S. Harriague, and K. Laßmann, "Computational experience showing the influence of the fuel-to-sheath thermal conductance in reactor fuel temperature predictions," Nuclear Engineering \& Design, vol. 73, no. 1, pp. 95-99, 1982.

[3] A. C. Marino and D. O. Brasnarof, "Modelling and design of advanced PHWR fuels," in Proceedings of the International Atomic Energy Agency Technical Meeting on "Pressurised Heavy Water Reactor (PHWR) Fuel Modelling”, Mumbai, India, December 2006.

[4] A. C. Marino and P. C. Florido, "High power ramping in commercial PHWR fuel at extended burnup," Nuclear Engineering \& Design, vol. 236, no. 13, pp. 1371-1383, 2006.

[5] E. Estévez, M. Markiewicz, and A. C. Marino, "Simulación del comportamiento se una Barra Combustible para el Reactor CAREM 25," in XXVII Reunión Científica de la Asociación Argentina de Tecnología Nuclear (AATN '00), Buenos Aires, Argentina, November 2000, paper 39.

[6] A. C. Marino, M. Markiewicz, and E. Estévez, "Verificación del Comportamiento de las Barras Combustibles para el Reactor CAREM vía Código de Cálculo para el Núcleo de Equilibrio," in XXVIII Reunión Científica de la Asociación Argentina de Tecnología Nuclear (AATN '10), Buenos Aires, Argentina, November 2010, paper 120.

[7] J. Killeen, V. Inozemtsev, and J. A. Turnbull, "Fuel modelling at extended burnup: IAEA coordinated research project FUMEX-II," in Proceedings of the International LWR Fuel Performance Meeting (Top Fuel '06), Salamanca, Spain, October 2006.

[8] A. C. Marino, E. J. Savino, and S. Harriague, "BACO (BArra COmbustible) code version 2.20: a thermo-mechanical description of a nuclear fuel rod," Journal of Nuclear Materials, vol. 229, pp. 155-168, 1996.

[9] "Fuel modelling at extended burnup," Report of the Co-ordinated Research Programme on Fuel Modelling at Extended Burnup-FUMEX-, 1993 1996, IAEA, IAEATECDOC-998.

[10] A. C. Marino, D. O. Brasnarof, G. L. Demarco et al., "CRP FUMEX II del OIEA: Validación de BaCo a Altos Quemados," in XXXIII Reunión Anual de la Asociación Argentina de Tecnología Nuclear (AATN '06), Buenos Aires, Argentina, November 2006.

[11] A. C. Marino and E. J. Savino, "Sensitivity analysis applied to nuclear fuel performance related to fabrication parameters and experiments," in Proceedings of the 14th International Conference on Structural Mechanics in Reactor Technology, Lyon, France, August 1997.

[12] A. C. Marino and P. C. Florido, "High power ramping in commercial PHWR fuel at extended burnup," Nuclear Engineering \& Design, vol. 236, no. 13, pp. 1371-1383, 2006.

[13] A. C. Marino, "Computer simulation of the behaviour and performance of a CANDU fuel rod," in Proceedings of the 5th
International Conference on CANDU Fuel, Toronto, Canada, September 1997.

[14] A. C. Marino and G. L. Demarco, "3D assessment of the cracked $\mathrm{UO}_{2}$ pellets behaviour," in Proceedings of the International LWR Fuel Performance Meeting (Top Fuel '06), Salamanca, Spain, October 2006.

[15] A. C. Marino, E. Pérez, and P. Adelfang, "Irradiation of Argentine (U,Pu) $\mathrm{O}_{2}$ MOX fuels. Post-irradiation results and experimental analysis with the BACO code," Journal of Nuclear Materials, vol. 229, pp. 169-186, 1996.

[16] A. C. Marino, "Crack and dishing evolution models and PCISCC considerations for fuel pellets in a quasi-bi-dimensional environment," in Proceedings of the International Seminar on Pellet-Clad Interaction in Water Reactor Fuels (PCI '04), Aixon-Provence, France, March 2004.

[17] A. C. Marino, "Bases para el desarrollo de códigos de comportamiento de barras combustibles nucleares," in XXVII Reunión Científica de la Asociación Argentina de Tecnología Nuclear (AATN '00), Buenos Aires, Argentina, November 2000, paper 145 .

[18] H. Freeborn et al., "Light water reactor fuel rod modelling code evaluation,” EPRI NP 369 (Project 397-1), Final report, March 1977.

[19] MATPRO-Version 11, "A handbook of material properties for use in the analysis of light water reactor fuel behaviour," NUREG/CR-0497 TREE-1280, February 1979.

[20] D. R. Olander, Fundamental Aspects of the Nuclear Fuel Reactor Elements, US Energy Research and Development Administration, 1976.

[21] I. Misfeld, "The D-COM blind problem on fission gas release," IAEA, International Working Group on Fuel Performance and Technology for Water Reactors, OECD-NEA-CSNI/IAEA Specialist's Meeting on Water Reactor Fuel Safety and Fission Products Release in Off-Normal and Accident Conditions, RISØ National Laboratory, IWGFTP/16 (1983).

[22] D. O. Brasnarof, A. C. Marino, P. C. Florido et al., "CARA development: an Argentinean fuel cycle challenge," in Proceedings of the 9th International Conference on CANDU Fuel "Fuelling a Clean Future", Ramada Inn on the Bay, Belleville, Canada, September 2005.

[23] "Status of advanced light water reactor designs, 2004," IAEATECDOC-CD-1391, May 2004.

[24] A. C. Marino, J. E. Bergallo, and F. Barrera, "Irradiación experimental de barras combustibles CARA en el Reactor OECD Halden," in XXVII Reunión Científica de la Asociación Argentina de Tecnología Nuclear (AATN '00), Buenos Aires, Argentina, November 2000, paper 68.

[25] J. E. Garcés, A. C. Marino, and G. Bozzolo, "Theoretical description of the interdiffusion of $\mathrm{Al}$ in the U-Mo solid solution," Applied Surface Science, vol. 219, no. 1-2, pp. 47-55, 2003.

[26] A. C. Marino, G. L. Demarco, and P. C. Florido, "An approach to the $3 \mathrm{D}$ modelling of the $\mathrm{UO}_{2}$ pellets behaviour under irradiation conditions," in Proceedings of the IAEA Technical Meeting on Fuel Behaviour Modelling Under Normal, Transient and Accident Conditions, and High Burnups, Kendal, UK, September 2005.

[27] A. C. Marino, "Probabilistic safety criteria on high burnup HWR fuels," in Proceedings of the IAEA's Technical Committee Meeting on "Technical and Economic Limits to Fuel Burnup Extension", Bariloche, Argentina, November 1999. 
[28] J. A. Turnbull, J. C. Killeen, and E. Sartori, "Experimental data on PCI and PCMI within the IFPE database," in Proceedings of the International Seminar on Pellet-Clad Interaction in Water Reactor Fuels (PCI '04), Aix-en-Provence, France, March 2004.

[29] A. C. Marino and E. J. Savino, "Applications of simple rules of fuel failure in a computer model simulation for nuclear fuel behaviour and performance," in Proceedings of the International Topical Meeting on Light Water Reactor Fuel Performance, pp. 165-172, Portland, Ore, USA, March 1997. 

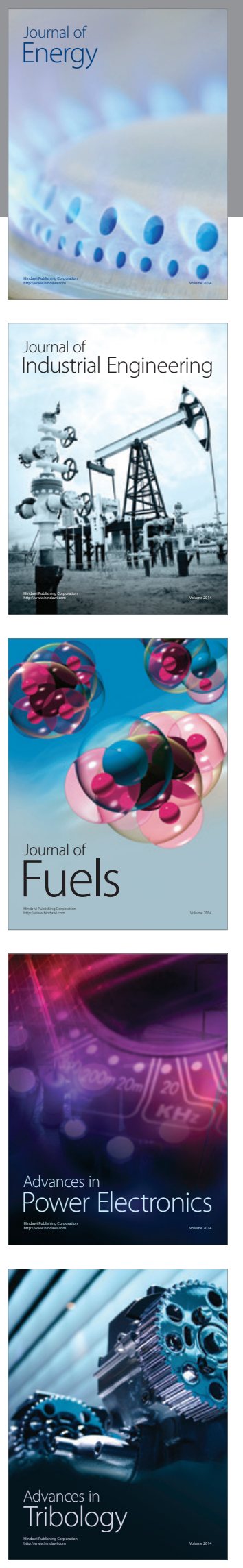
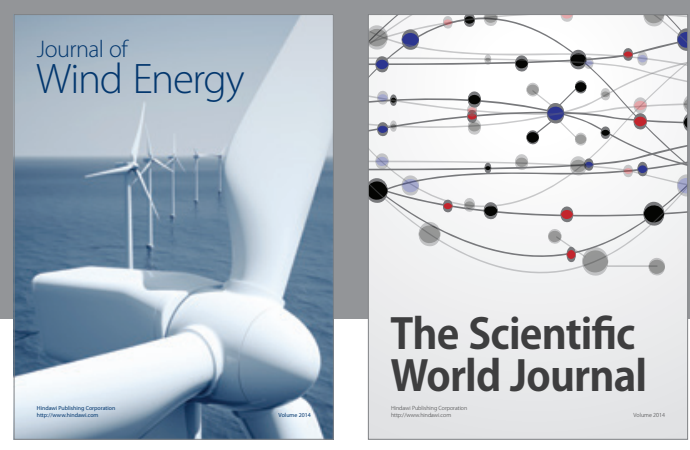

The Scientific World Journal

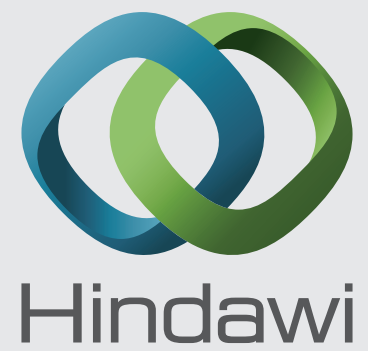

Submit your manuscripts at http://www.hindawi.com
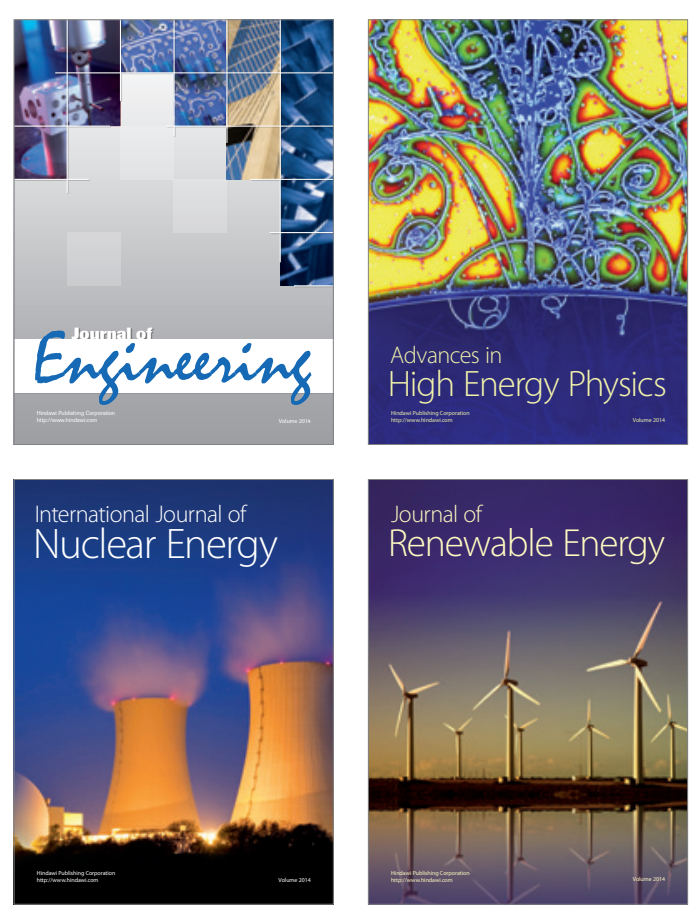

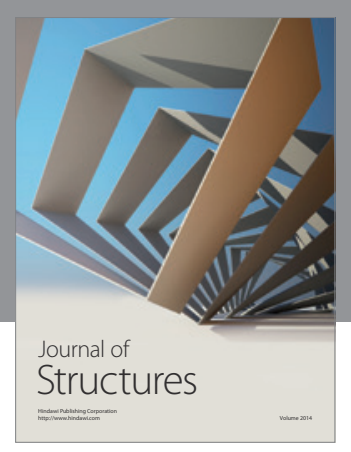

Rotating
Mechinery
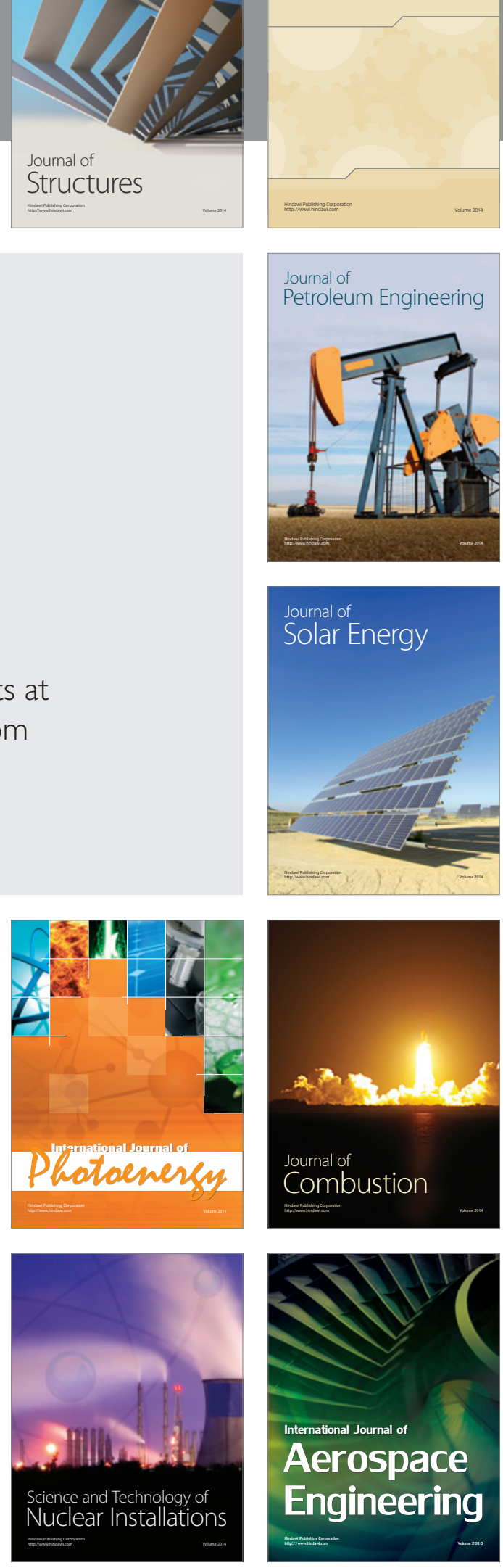